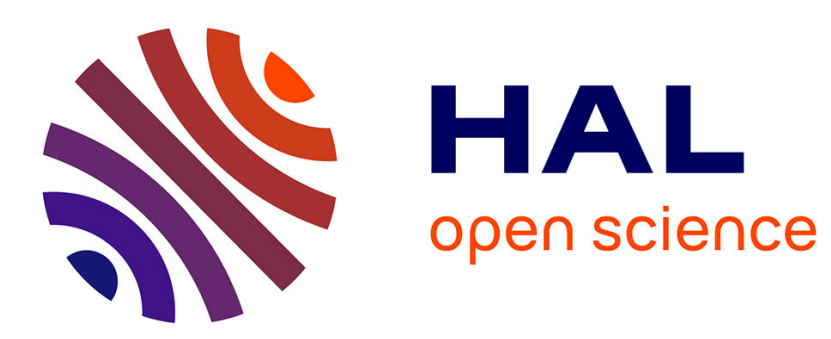

\title{
An effective streamflow process model for optimal reservoir operation using stochastic dual dynamic programming
}

\author{
L. Raso, P.O. Malaterre, J.C. Bader
}

\section{- To cite this version:}

L. Raso, P.O. Malaterre, J.C. Bader. An effective streamflow process model for optimal reservoir operation using stochastic dual dynamic programming. Journal of Water Resources Planning and Management, 2017, 143 (4), pp.11. 10.1061/(ASCE)WR.1943-5452.0000746 · hal-01761533

\section{HAL Id: hal-01761533 \\ https://hal.science/hal-01761533}

Submitted on 9 Apr 2018

HAL is a multi-disciplinary open access archive for the deposit and dissemination of scientific research documents, whether they are published or not. The documents may come from teaching and research institutions in France or abroad, or from public or private research centers.
L'archive ouverte pluridisciplinaire HAL, est destinée au dépôt et à la diffusion de documents scientifiques de niveau recherche, publiés ou non, émanant des établissements d'enseignement et de recherche français ou étrangers, des laboratoires publics ou privés. 


\section{An effective streamflow process model for optimal 2 reservoir operation using Stochastic Dual Dynamic Programming}

Luciano Raso $^{1}$, Pierre-Olivier Malaterre ${ }^{1}$, and Jean-Claude Bader ${ }^{2}$

Corresponding author: Luciano Raso, IRSTEA, Montpellier, France. (luciano.raso@irstea.fr)

${ }^{1}$ IRSTEA, UMR-GEAU, Montpellier,

France.

${ }^{2}$ IRD, UMR-GEAU, Montpellier, France. 
${ }_{4}$ Abstract. We present an innovative streamflow process model to be used

${ }_{5}$ for reservoir operational rule design in Stochastic Dual Dynamic Program-

6 ming (SDDP). Model features, which can be applied independently, are: i)

7 a non-linear multiplicative process model for the forward phase, which pro-

s duces positive streamflow values only, and its linearized version for the back-

9 ward phase, and ii) a non-uniform time-step, which divides the hydrologi-

${ }_{10}$ cal period in time-steps of different length in order to have a process with

${ }_{11}$ approximately constant variance. Model identification is straightforward as

${ }_{12}$ for additive periodic autoregressive model generally used in SDDP. We ap-

${ }_{13}$ plied this model on the Senegal River for the optimal operation of Manan-

${ }_{14}$ tali reservoir, and evaluated the proposed solutions against streamflow pro-

${ }_{15}$ cess model currently used in the water management literature. 


\section{Introduction}

In reservoir operation, present benefits must be balanced with future, uncertain ones

${ }_{17}$ [Soncini-Sessa et al., 2007; Castelletti et al., 2008]. After each release decision, new infor-

18 mation becomes available and partially reduces uncertainty. Optimal reservoir operation

${ }_{19}$ can be framed as a Multistage Stochastic Programming problem [Birge and Louveaux,

20 1997; Shapiro and Andrzej, 2003], which, for long horizon, is conveniently solved by

${ }_{21}$ Stochastic Dynamic Programming [Bellman and Dreyfus, 1966]. SDP, notwithstanding

22 its elegance and potential, is affected by the so-called "curse of dimensionality", limiting

${ }_{23}$ its application to systems made of few variables [Stedinger et al., 1984; Trezos and Yeh, 1987]. In the literature, some alternatives try to circumvent these limitations, for example

${ }_{25}$ by defining an optimal trajectory [Turgeon, 1980] or fixing the release policy family and

${ }_{26}$ find the parameters by evolutionary algorithm [Nicklow et al., 2009; Reed et al., 2013].

${ }_{27}$ These solutions, even if advantageous for some aspects, have rarely been tested over large [Pereira and Pinto, 1991] (SDDP) is an approximation of SDP that largely attenuates the

so curse of dimensionality. SDDP, however, requires the optimization problem to be modeled

31 as linear, since problem linearity ensures cost-to-go function convexity.

SDDP requires identifying a linear stochastic inflow model which reproduces the stream-

${ }_{33}$ flow process and its uncertainty. Streamflow process model identification is a critical step

34 in dynamic programming problem setting, sometimes referred to as "curse of modelling"

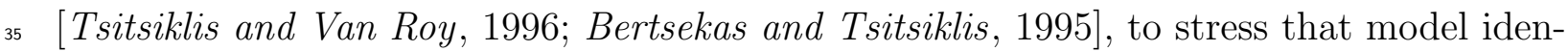

${ }_{36}$ tification can be problematic, hence a limitation for the methodology. SDDP applications 
Author-produced version of the article published in Journal of Irrigation and Drainage Engineering-ASCE, 2017, N¹43(4)

The original publication is available at http://ascelibrary.org

Doi: 10.1061/(ASCE)WR.1943-5452.00007466

$\mathrm{X}-4$

RASO ET AL.: EFFECTIVE STREAMFLOW PROCESS MODEL FOR SDDP

37 generally use a standardized Periodic Autoregressive (PAR) model of lag 1 [ Tilmant et al.,

${ }_{38}$ 2008; Tilmant and Kelman, 2007; Tilmant et al., 2007, 2010, 2012; Tilmant and Kinzel-

39 bach, 2012; Tilmant et al., 2009; Goor et al., 2010; Arjoon et al., 2014; Marques and

40 Tilmant, 2013; Gjelsvik et al., 2010], also known as Thomas-Fiering model [Loucks, 1992].

${ }_{41}$ The drawback of this additive model is the non-neglegible probability of negative dis-

${ }_{42}$ charge values. This is to be avoided, because negative discharges have no physical sense.

${ }_{43}$ Existing solutions dealing with negative discharges [Stedinger and Taylor, 1982; Pereira

44 et al., 1984; Bezerra et al., 2012] use non-linear transformations that make these models

45 not usable in SDDP.

${ }_{46}$ The monthly time-step preserves the process as markov, but it risks to underestimate

${ }_{47}$ the system adaptivity to changing conditions. Such a large time-step, in fact, may not

48 take into account the adaptivity at a smaller time-step, and it can be a limitation to the

49 analysis of system response to fast processes, such as flood, resulting in an underestimation

so of system capacity to react to this type of events.

${ }_{51} \quad$ Short-term system adaptation can be taken into account by a time decomposition ap-

${ }_{52}$ proach [Karamouz et al., 2003]. In time decomposition, long-term policies are refined by

53 optimizations at shorter-term windows, using results from the long-term optimization as

${ }_{54}$ boundary conditions. Even if time decomposition increases the accuracy of performance

${ }_{55}$ estimation, short-term optimization can use more information than the long-term opti-

${ }_{56}$ mization supposes, leading to an underestimation of the performance value in the long

${ }_{57}$ term planning [Weijs, 2011]. Therefore, aggregating discharges at large time steps is

${ }_{58}$ an approximation with negative impact on performance, and time-step length must be a

${ }_{59}$ trade-off between i) calculation time and capacity to represent the process as markov, and 
ii) accurate representation of the relevant processes. The former requires a long time-step,

61

62

63

${ }_{66}$ a relatively slow process compared to flood.

\section{Methodology}

79

80 

control problem.

$$
\begin{aligned}
& \text { Find } \pi_{t}, \forall t \in\{1, \ldots, H\}: \\
& \qquad \max _{\pi_{t}} \sum_{t=1}^{H} \underset{\mathbf{q}_{t}}{\mathbb{E}}\left[g_{t}\left(\mathbf{v}_{t}, \mathbf{r}_{t}, \mathbf{q}_{t}\right)\right]
\end{aligned}
$$

Subject to:

$$
\begin{gathered}
\mathbf{v}_{t}=\mathbf{v}_{t-1}+\Delta t \cdot\left(I \cdot\left[\mathbf{r}_{t}, \mathbf{q}_{t}\right]-O \cdot\left[\mathbf{r}_{t}, \mathbf{q}_{t}\right]\right) \\
\mathbf{c}_{t}\left(\mathbf{v}_{t}, \mathbf{r}_{t}, \mathbf{q}_{t}\right) \leq 0 \\
\mathbf{q}_{t} \sim f_{\mathbf{Q}_{t}}
\end{gathered}
$$

${ }_{84}$ In Problem (1), vectors $\mathbf{v}_{t} \in \mathbb{R}^{N_{\text {res }}}, \mathbf{r}_{t} \in \mathbb{R}^{N_{\text {dec }}}, \mathbf{q}_{t} \in \mathbb{R}^{N_{\text {scen }}}$ represent reservoir volumes,

${ }_{85}$ discharge decisions, and scenarios, at instant $t$ for stocks and in the period $[t-\Delta t, t)$ for

${ }_{86}$ flows; $\pi_{t}$ is the optimal release rule, that suggests the optimal release decision $\mathbf{r}_{t}$ in function ${ }_{87}$ of the occurring scenario, i.e. the realization of $\mathbf{q}_{t}$. In Expression $(1 \mathrm{~b}), g_{t}(\cdot)$ is a $\mathbb{R}^{N}$ to $\mathbb{R}$ ${ }_{\text {s8 }}$ function, representing the system objective at $t$, where $N=N_{\text {res }}+N_{\text {dec }}+N_{\text {scen }}$. Equation

${ }_{89}$ (1c) is the continuity equation, represented by the reservoirs mass balance. In Equation

${ }_{90}$ (1c), $\Delta t$ is time-step length, $I$ and $O$ are the input and output matrix, of dimension $N_{\text {res }} \times$ ${ }_{91}\left(N_{\text {dec }}+N_{\text {scen }}\right)$, associating at each inflow and discharge decision to its reservoir. $O(i, j)$ ${ }_{92}$ and $I(i, j)$ is 1 if the $i$ variable is input or output of reservoir $j, 0$ elsewhere. Scenarios ${ }_{93} q_{t}$, in Expressions (1e), are either stochastic or deterministic scenarios. Deterministic ${ }_{94}$ scenarios are a vector of values, stochastic scenarios are random variables distributed as ${ }_{95} f_{\mathbf{Q}_{t}}\left(\mathbf{q}_{t}\right)$. Future inflows to the reservoirs are described as stochastic scenarios, while other 
96 variables, such as evaporation, for which uncertainty can be neglected, are considered as

$$
\begin{array}{r}
\max _{\mathbf{r}_{1}} g_{1}\left(\mathbf{v}_{1}, \mathbf{r}_{1}, \mathbf{q}_{1}\right)+\underset{q_{2, i}}{\mathbb{E}}\left[\max _{\mathbf{r}_{2, i}} g_{2}\left(\mathbf{v}_{2, i}, \mathbf{r}_{2, i}, \mathbf{q}_{2, i}\right)+\underset{q_{3, i}}{\mathbb{E}}[\ldots\right. \\
\left.\left.\ldots+\underset{q_{H, i}}{\mathbb{E}}\left[\max _{\mathbf{r}_{H, i}} g\left(\mathbf{v}_{H, i}, \mathbf{r}_{H, i}, \mathbf{q}_{H, i}\right)\right] \ldots\right]\right]
\end{array}
$$

under conditions given by Equations $(1 \mathrm{c}, ? ?, 1 \mathrm{~d})$, and with an initial condition $\mathbf{v}_{0}$. In some case, a condition on final time-step $\mathbf{c}_{H}\left(\mathbf{v}_{H}\right) \leq \mathbf{b}_{H}$ may be present, in the form of hard or soft constraint [van Overloop et al., 2008].

By solving Problem (2), the optimization procedure finds an optimal release rule for a future horizon $H$. In expression (2), the release rule $\left\{\pi_{t}\right\}_{t=1}^{H}$ is a decision tree, $\mathbf{r}_{t, i} \forall t, \forall i$, made of multiple bifurcations at each time-steps, $t \in[1: H]$, representing the optimal decision strategy adapted to the $i$ realisation of the stochastic variable, $\mathbf{q}_{t, i}$. The main drawback of Multistage Stochastic Programming is its computational complexity, which increases exponentially with $H$. Multistage Stochastic Programming can be applied when 
$H$ is small, for example in short term management [Raso et al., 2014]. In same case the problem has been aggregated and reduced to seasonal decisions [Seifi and Hipel, 2001]. For long-term optimization, however, MSP can be considered as a theoretical, rather than practical, method [Mayne et al., 2000].

Stochastic Dynamic Programming (SDP) decomposes the MSP problem in step-bystep optimal decision problems. Then, the optimization problem in Expression (2) can be written as Bellman Chain, as in Equation (3). SDP can solve problems with a much longer horizon, because problem complexity increases only linearly with $H$.

$$
F_{t}\left(\mathbf{v}_{t}, \mathbf{q}_{t}\right)=\max _{\mathbf{r}_{t}} g_{t}\left(\mathbf{v}_{t}, \mathbf{r}_{t}, \mathbf{q}_{t}\right)+\underset{\mathbf{q}_{t+1}}{\mathbb{E}}\left[F_{t+1}\left(\mathbf{v}_{t+1}, \mathbf{q}_{t+1}\right)\right]
$$

${ }_{125}$ In Equation (3), $F_{t}$ is the cost-to-go function, the average cost for leaving the system in the state $\left[\mathbf{v}_{t}, \mathbf{q}_{t}\right]$, which is the compromise of present and future benefits, $g_{t}(\cdot)$ and $F_{t+1}$. In SDP, Equation (3) is the release rule $\pi_{t}$, which maps the system state to the optimal release decisions.

Equation (3) can be solved backwards, from $t=H$ to the initial time-step. Condition (1e) is substituted by Condition (4). The probability transition from $\mathbf{q}_{t-1}$ to $\mathbf{q}_{t}$, together with the continuity Equation (1c), makes up the transition equations, which describes the system dynamic from one state to the next one.

SDP requires the stochastic transition to be expressed as a Markov process [Rabiner and Juang, 1986], i.e. the probability of each event, $f\left(\mathbf{q}_{t}\right)$, depends only on the state at previous instant, $\mathbf{q}_{t-1}$, as in Equation (4).

$$
\mathbf{q}_{t} \sim f_{Q_{t}}\left(\mathbf{q}_{t} \mid \mathbf{q}_{t-1}\right)
$$




\subsection{Multiplicative error model identification}


In this section we propose a multiplicative error model to deal with the problem of generating negative discharges.

In SDDP, the stochastic hydrological model must be linear, but no conditions are imposed on distribution $f_{t}\left(\mathbf{q}_{t}\right)$. There is not specific reason to prefer separate additive terms for the predictive and the uncertain parts of the model [Koutsoyiannis, 2009]. In the following, we show a procedure to estimate a linear model in $\mathbf{q}_{t}$, with a multiplicative stochastic component.

We start from a multivariate signal of observed discharge, which we want to reproduce, $\mathbf{q}_{t}$, made of $T$ samples for $N_{y}$ years of data, where $T$ is the number of time-steps per year. The original signal is transformed into $\mathbf{y}_{t}$ according to Equation (5).

$$
\mathbf{y}_{t}=\log \left(\mathbf{q}_{t}\right)-\log \left(\overline{\mathbf{q}}_{\tau}\right)
$$

In Equation (5), $\tau=1, \ldots, T$ is the periodic time index. Equation (6) defines $\overline{\mathbf{q}}_{\tau}$, i.e. the periodic geometric average of $\mathbf{q}_{t}$ for all $\mathbf{t}=\tau$, where $\mathbf{t}=(t-1) \bmod T+1$, and $\bmod$ is the modulus (or remainder) operator.

$$
\overline{\mathbf{q}}_{\tau}=\left(\prod_{\mathbf{t}}^{N_{y}} \mathbf{q}_{t}\right)^{1 / N_{y}}=\exp \left(\frac{1}{N_{y}} \sum_{\mathbf{t}}^{N_{y}} \log \mathbf{q}_{t}\right), \forall \mathbf{t}=\tau
$$

Logarithm smooths extremes and long tails, making model identification easier. In fact, logarithm is a particular case of the Box-Cox transformation, suggested in Box et al. [1970] to deal with non-normal residuals. We use the $\mathbf{y}_{t}$ signal to identify an ARMA model on the $N_{\text {stoch }}$ dimensional signal. Contemporaneous ARMA (CARMA) [Camacho et al., 1987] are an effective sub-class of multivariate ARMA model that proved effective in hydrological applications. A CARMA model is identified as a set of $N_{\text {stoch }}$ univariate 

$\xi_{t-j}=\frac{q_{t-j}}{\hat{q}_{t-j}}$.

$$
y_{t}=\sum_{i=1}^{p} \phi_{\tau, i} y_{t-i}+\sum_{j=1}^{q} \psi_{\tau, i} \varepsilon_{t-i}+\varepsilon_{t}
$$

In Equation $(7), \varepsilon_{t}$ is the stochastic process, extracted from $\mathcal{N}\left(0, \sigma_{\tau}^{2}\right)$, independent on previous $\varepsilon_{t-i}$, where $\varepsilon_{t-i}=y_{t-i}-\hat{y}_{t-i} ; y_{t}$ is observed value, and $\hat{y}_{t}$ is expected value of Model (7), i.e. when $\varepsilon_{t}=0$. Identifying a $P A R M A_{\tau}(p, q)$ model is defining parameters $\phi_{\tau, i}, \psi_{\tau, i}, \sigma_{\tau}$ of Equation (7) $\forall i, \forall j, \forall \tau$.

Equation (7) can be written as transition from $q_{t-i}$ to $q_{t}$. By inversion of Equation (5) and some rearrangement, one can obtain Equation (8).

$$
q_{t}=\alpha_{\tau} \cdot \prod_{i=1}^{p} q_{t-i}{ }^{\phi_{\tau, i}} \cdot \prod_{j=1}^{q} \xi_{t-i} \psi_{\tau, j} \cdot \xi_{t}
$$

where $\xi_{t} \sim \ln \mathcal{N}\left(0, \sigma_{\tau}\right), \alpha_{\tau}=\bar{q}_{\tau} / \prod_{i=1}^{p} \bar{q}_{\tau-i}^{\phi_{\tau, i}}$, with $\bar{q}_{\tau}$ defined as in Equation (6), and

If in model (7) residuals are normal and additive, in model (8) residuals become lognormal and multiplicative. For positive initial condition, $q_{0}>0$, multiplicative random 
process ensures non-negative values of inflow process, offering a better representation of the hydrological process.

In Model (8), dependencies on $q_{t-i}$ are non-linear. Model (8) can be directly employed in the forward phase of SDDP, also using a less parsimonious model than in backwards, which can produce, in some cases, considerably better results [Bartolini and Salas, 1993]. SDDP forward phase requires in fact a streamflow time series, regardless of the method to generate it. In backward phase, instead, SDDP must solve a linear optimization, therefore transition from $q_{t-1}$ to $q_{t}$ must be linear. Model (8), to be applied in backward phase of SDDP, must be written as in Equation (9), which is Model (8) linearized by taylor expansion on the argument median, i.e. $q_{t-i}=\bar{q}_{\tau-i}$ and $\xi_{\tau-j}=1, \forall \tau, \forall i, \forall j$.

$$
q_{t}=\left[\sum_{i=1}^{p} \rho_{\tau, i} q_{t-i}+\sum_{j=1}^{q} \omega_{\tau, i} \xi_{t-i}+\kappa_{\tau}\right] \cdot \xi_{\tau}
$$

where parameters are defined in Equations (10) and derived in Appendix A.

$$
\begin{aligned}
\rho_{\tau, i} & =\phi_{\tau, i} \cdot \frac{\bar{q}_{\tau}}{\bar{q}_{\tau-i}} \\
\omega_{\tau, i} & =\psi_{\tau, i} \cdot \bar{q}_{\tau} \\
\kappa_{\tau} & =\bar{q}_{\tau} \cdot\left(1-\sum_{i=1}^{p} \phi_{\tau, i}-\sum_{j=1}^{q} \psi_{\tau, i}\right)
\end{aligned}
$$

${ }_{204}$ Linearisation introduces an approximation error that must be quantified. Equation (11)

defines $e_{t}$, the error due to linearisation.

$$
e_{t}=q_{t}^{\operatorname{lin}}-q_{t}^{\mathrm{nl}}
$$

where $q_{t}^{\text {lin }}$ and $q_{t}^{\mathrm{nl}}$ are the output of Model (9) and Model (8) for $\xi_{t}=1$. Considering, for simplicity of notation, an univariate Multiplicative Periodic Autoregressive Model, 


$$
\begin{aligned}
\mathbb{E}\left(e_{\tau}\right) & =\int_{0}^{+\infty} e_{t}\left(q_{t-1}\right) \cdot f_{\tau}\left(q_{t-1}\right) \cdot d q_{t-1} \\
q_{p}\left(e_{\tau}\right) & =e_{t}\left(F_{q_{\tau-1}}^{-1}(p)\right)
\end{aligned}
$$

${ }^{211}$ Where $q_{p}\left(e_{\tau}\right)$, is the $p$ quantile of $e_{\tau}$, and $F_{q_{\tau-1}}(\cdot)$ is the Cumulative Density Function ${ }_{212}$ of $q_{\tau-1}$. Distribution of $q_{\tau-1}$ can be obtained from $f\left(y_{\tau-1}\right)$ through Equation (5). From ${ }_{218}(10 \mathrm{c})$.

\subsection{Non-uniform time-step length}


period in time-steps of different length in order to maintain an approximately homogeneous variance.

In the following, we describe the proposed procedure to select the non-uniform time-step length, $\Delta k(\tau)$, to aggregate data from the finest time-step, $\tau_{d}=1, \ldots, T_{d}$ to the desired level of aggregation, $\tau=1, \ldots, T$, such that the aggregated time-step is $\Delta \tau=\Delta \tau_{d} \cdot \Delta k$.

Starting from the data at the finest time aggregation of period $T_{d}$ and time index $\tau_{d}=1, \ldots, T_{d}$, we identify a $\operatorname{PAR}_{T_{d}}(1)$ model on $q_{t}$, having parameters $\phi_{\tau_{d}}, \sigma_{\tau_{d}}^{2}, \forall \tau_{d} \in$ $\left\{1, \ldots, T_{d}\right\}$, defined as in Equation (7). Even if such simple model may be not accurate enough for prediction purposes, it is generally sufficient to catch the dominant dynamics.

Time-steps of homogeneous variability, $\Delta k(\tau)$, must be such that $V A R\left(q_{\tau_{0}+\Delta k(\tau)} \mid q_{\tau_{0}}\right)$ is approximately homogeneous for all $\tau$, and $\sum \Delta k(\tau)=T_{d}$. Equation (13) defines variance of $q_{\tau_{0}+\Delta k(\tau)}$ conditional to $q_{\tau_{0}}$ as function of time-step residual variance, $\sigma_{\tau_{d}}^{2}$.

$$
V A R\left(q_{\tau_{0}+\Delta k(\tau)} \mid q_{\tau_{0}}\right)=\sum_{\tau_{d}=\tau_{0}+1}^{\tau_{0}+\Delta k(\tau)} \prod_{i=\tau_{d}+1}^{\tau_{0}+\Delta k(\tau)} \phi_{i}^{2} \cdot \sigma_{\tau_{d}}^{2}
$$

where, by convention, $\phi_{i}^{2}=1$ if $i>\tau_{0}+\Delta k(\tau)$.

In Equation (13), $\phi_{\tau_{d}}$ is generally close to one, especially for $\tau_{d}$ where variability is small, and time-steps $\Delta k$ can be larger. Considering $\prod \phi_{\tau_{d}}^{2}$ in Equation (13) as equal to one, $V A R\left(q_{\tau_{0}+\Delta k_{i}} \mid q_{\tau_{0}}\right)$ can be written as proportional to the sum of variances only, which we take as indicator of variability. This allows us to define a cumulated variability that depends on $\tau_{d}$ only.

The residual variance is used to define a Cumulative Variability in function of $\tau$, as in Equation (14). 
In Equation (14), the numerator is the cumulative variance until $\tau_{d}$, the denominator is

$$
C V\left(\tau_{d}\right)=\frac{\sum_{t=1}^{\tau_{d}} \underline{\sigma}_{\tau_{d}}^{2}}{\sum_{t=1}^{T_{d}} \underline{\sigma}_{\tau_{d}}^{2}}
$$

one. The non-uniform time-step $\Delta k(\tau)$ is then chosen by splitting the hydrological period

${ }_{24}$ in $T$ time-steps having approximately homogenous variability, as in Equation (15).

$$
\Delta k(\tau)=\left\lfloor C V^{-1}\left(\frac{\tau}{T}\right)-C V^{-1}\left(\frac{\tau-1}{T}\right)\right\rceil ; \forall \tau=\{1, \cdots, T\}
$$

${ }_{249}$ where operator $\left\lfloor\cdot 7\right.$ returns the nearest integer. By convention, $C V^{-1}(0)=0$.

The discharge signal is aggregated using the variable time-step, and a model is identified on the aggregated signal according to procedure in Section 2.1, or others.

\section{Application to the Senegal river}

The Senegal River, West Africa, is a $1790 \mathrm{~km}$ long river. Its drainage basin extension ${ }_{253}$ is $270.000 \mathrm{~km}^{2}$, over Guinea, Mali, Senegal and Mauritania. The river inflow is extremely ${ }_{254}$ variable, following the tropical raining seasonality with a marked difference between the 
Author-produced version of the article published in Journal of Irrigation and Drainage Engineering-ASCE, 2017, $\mathrm{N}^{\circ} 143(4)$

The original publication is available at http://ascelibrary.org

$\mathrm{X}-16$

RASO ET AL.: EFFECTIVE STREAMFLOW PROCESS MODEL FOR SDDP 
${ }_{285}$ (May 15) and 285 (October 12). 
Author-produced version of the article published in Journal of Irrigation and Drainage Engineering-ASCE, 2017, N¹43(4)

The original publication is available at http://ascelibrary.org

$\mathrm{X}-18$

RASO ET AL.: EFFECTIVE STREAMFLOW PROCESS MODEL FOR SDDP

${ }_{307}$ band, for most of the lags larger than zero. This confirms the validity of the non-uniform 


\subsection{Effects of non-uniform aggregation on reservoir operation}




$$
\begin{aligned}
& v_{\text {min }} \leq v_{t} \leq v_{\text {max }} \\
& 0 \leq r_{\text {turb }, t} \leq r_{\text {turb,max }} \\
& r_{\text {spill }, t} \geq m_{\text {safety }} \cdot\left(v_{t}-v_{\text {safety }}\right)
\end{aligned}
$$

Inequalities (16a) and (16b) are physical constraints, derived from the system characteristics. Inequality (16c) is a legal condition that forces to draw down the reservoir when its volume exceeds the safety threshold, $v_{\text {safety }}$. Constraint on release through spillages is large enough for never being active during the simulation period, as verified a posteriori, and therefore it is not included. Inequalities (16b) and (16c) are implemented as hard constraint, inequality (16a) is implemented as soft constraint to avoid non-feasibility.

The system objective is the yearly average energy production $J_{E}=1 / N_{\text {years }} \sum_{t=1}^{T \times N_{\text {years }}} E_{t}$, composed of the sum of the daily energy production, $E_{t}$, as defined in Equation (17), for the entire simulation horizon $T \times N_{\text {years }}$, where $N_{\text {years }}=43$, from 1970 to 2012 . 
In Equation (17), $\Delta h_{t}$ is the hydraulic head $[m], r_{\text {turb }, t}$ is discharge trough turbines $\left[\mathrm{m}^{3} / \mathrm{s}\right] . \eta$ is a multiplicative factor, such that $\eta=\rho \cdot g \cdot \widehat{\eta}\left(r_{t}, \Delta h\right) \cdot 24 \cdot 10^{-6}$, where $\rho$ is the water density, $1000[\mathrm{~kg} / \mathrm{m} 3], g$ is the gravity acceleration, $9.8\left[\mathrm{~m} / \mathrm{s}^{2}\right], \widehat{\eta}$ is the efficiency coefficient, considered equal to $0.9,24$ and $10^{-6}$ are unit transformation coefficients, $[h / d]$ 375 and $[M W / W] . E_{t}$ is expressed in $[M W h]$.

Equation (17), to be employed in linear optimization within SDDP, is approximated by expressing it as linear function of $r_{t}$ and $v_{t}$, linearized at an operational point, under the

$$
E_{t}=\eta \cdot \Delta k(\tau) \cdot \Delta h_{t} \cdot r_{\mathrm{turb}, t}
$$

hypothesis of cylindric reservoir. Equation (18) defines the operational time-step objective 79 indicator used in SDDP, being the weighted sum of releases and volume.

$$
\begin{aligned}
E_{t}^{\mathrm{op}}=E_{0, t}+ & \\
+\eta \cdot \Delta k(\tau) \cdot\{ & +\left[\frac{r_{\text {turb }, 0}}{A_{0}}\right] \cdot v_{t} \\
& +\left[h_{0}-h_{0}^{v}-m_{v}\left(1+r_{\text {turb }, 0}\right)\right] \cdot r_{\text {turb }, t} \\
& \left.+\left[-m_{v}\right] \cdot r_{\text {spill }, t}\right\}
\end{aligned}
$$

In Equation (18), $E_{0, t}=\eta \cdot \Delta k(\tau) \cdot\left\{\left[h_{0}-h_{0}^{v}\right] \cdot r_{\text {turb }, 0}-\left[\frac{r_{\text {turb }, 0}}{A_{0}}\right] \cdot v_{0}-\left[h_{0}-h_{0}^{v}-\right.\right.$ $\left.\left.{ }_{381} m_{v}\left(1+r_{\text {turb }, 0}\right)\right] \cdot r_{\text {turb }, 0}+m_{v} \cdot r_{\text {spill, }, 0}\right\}$. Parameters of Equation (18) and their derivation 382 are described in Appendix B.

Equation (17) is linearized at a normal operational point, that is the reservoir state 384 at which the reservoir is mostly operated, either historically observed or deduced from 385 system characteristics. We infer the operational point from the reservoir characteristics, 
Author-produced version of the article published in Journal of Irrigation and Drainage Engineering-ASCE, 2017, N¹43(4)

The original publication is available at http://ascelibrary.org

X - 22

Doi: 10.1061/(ASCE)WR.1943-5452.00007466

considering $v_{0}=v_{\text {safety }}$, i.e. the safety limit, $r_{t u r b, 0}=\mathbb{E}\left(q_{t}\right)$, i.e. the average inflow to the reservoir, and $r_{\text {spill }, 0}=0 \mathrm{~m}^{3} / \mathrm{s}$, which considers no release through the spillages.

The optimization is performed using 25 extractions for the forward phase and 25 for the backward one until convergence, attained at an accuracy level of $2 \times 10^{7}$. This accuracy level lays within the $\pm 2 \sigma$ of forward simulation results. We can consider with sufficient confidence that the algorithm has converged to the optimum.

Performance results show a moderate improvement for non-uniform aggregation. Optimal solution gives $J_{E}$ equal to $930 \mathrm{GWh} /$ year for the monthly aggregation, and 945 GWh/year in the non-uniform case, equivalent to an improvement of one week of average energy production.

We analyse the reservoir operation behaviour. Figure 10 shows the reservoir volume and the release trough turbines for the monthly and the non-uniform aggregation in response to the 2005 inflow scenario. Inflow peak is larger than maximum discharge trough turbines; therefore, to avoid spillage, the reservoir must be drawn down before the high flow period, in order to create a buffer that stores part of the incoming water. SDDP optimal operating rules are the results of an optimal compromise between the objectives of keeping a high water level and avoiding spillages.

Figure 10 plot (b) shows how the reservoir operation using non-uniform steps adjusts decisions at higher frequency during high uncertainty periods, adapting more rapidly to the new observed discharge. Thanks to this rapid adaptation during the high discharge period, reservoir operation using non-uniform aggregation can draw down the reservoir less, as shown in Figure 10 plot (a). 


\section{Conclusions and Discussion}


using non-uniform time-steps has a relatively homogeneous variance. This brings in a practical advantage: the non-uniform time-steps follow closer the changing hydrological variability along the year, adapting the decision more frequently during high variability periods, resulting in enhanced system performance evaluation. If time-step aggregation is sufficiently fine, a non-uniform aggregation may even make time decomposition needless.

Model linearization, used in the backward phase, introduces an error. The analysis on error due to linearisation show that the error average is negligible, growing to about $5 \%-10 \%$ of median discharge at 5\%-95\% quantiles of $q_{\tau-1}$, which we consider satisfactory; this depends, however, on the specific test-case and, a priori, we cannot exclude it to be a limitation for this model.

\section{Appendix A: Linear model parameters derivation}

Parameters $\rho_{\tau, i}, \omega_{\tau, i}, \kappa_{\tau}$ are derived by linerization of Model (8) on the median of its deterministic inputs, at $q_{t-i}=\bar{q}_{\tau-i}$, with $\bar{q}_{\tau}$ defined in Equation (6), and $\xi_{t-j}=1$.

Equation A1 is model (8) for $\xi=1$ written as Taylor expansion on its deterministic inputs.

$$
\begin{aligned}
q_{t}^{\mathrm{nl}} \approx & \sum_{i=1}^{p} \frac{\partial q_{t}^{\mathrm{nl}}}{\partial q_{t-i}} \cdot\left(q_{t-i}^{\mathrm{nl}}-\bar{q}_{t-i}\right)+ \\
& \sum_{\substack{j=1 \\
q}} \frac{\partial q_{t}^{\mathrm{nl}}}{\partial \xi_{t-j}}\left(\xi_{t-j}-\bar{\xi}_{t-j}\right)+ \\
& q_{t}^{\mathrm{nl}}\left(q_{t-i}, \xi_{t-j}\right)
\end{aligned}
$$




$$
\begin{aligned}
\frac{\partial q_{t}^{\mathrm{nl}}}{\partial q_{t-i}}= & \alpha_{\tau} \cdot\left[\phi_{\tau, i} \cdot q_{t-i}{ }^{\left(\phi_{\tau, i}-1\right)} .\right. \\
& \left.\prod_{k \in\{1, \ldots, p\} \backslash i} q_{t-k} \phi_{\tau, k} \cdot \prod_{j=1}^{q} \xi_{t-j} \psi_{\tau, i}\right] \\
\frac{\partial q_{t}^{\mathrm{nl}}}{\partial \xi_{t-j}}= & \alpha_{\tau} \cdot\left[\psi \cdot \xi_{t-j}\left(\psi_{\tau, j}-1\right) .\right. \\
& \left.\prod_{i}^{p} q_{t-i}^{\phi_{\tau, i}} \cdot \prod_{k \in\{1, \ldots, q\} \backslash j} \xi_{t-k} \psi_{\tau, k}\right]
\end{aligned}
$$

$$
\begin{aligned}
\rho_{\tau, i} & =\frac{\partial q_{t}^{\mathrm{nl}}}{\partial q_{t-i}}\left(\bar{q}_{t-i}, \bar{\xi}_{t-j}\right)= \\
& =\frac{\bar{q}_{\tau}}{\prod_{i=1}^{p} \bar{q}_{\tau-i}^{\phi_{\tau, i}}} \cdot\left[\phi_{\tau, i} \cdot \frac{\bar{q}_{t-i}^{\phi_{\tau, i}}}{\bar{q}_{t-i}} \cdot \prod_{k \in\{1, \ldots, p\} \backslash i} \bar{q}_{t-k}^{\phi_{\tau, k}}\right]= \\
& =\phi_{\tau, i} \cdot \frac{\bar{q}_{\tau}}{\bar{q}_{\tau-i}} \\
\omega_{\tau, j} & =\frac{\partial q_{t}^{\mathrm{nl}}}{\partial \xi_{t-j}}\left(\bar{q}_{t-i}, \bar{\xi}_{t-j}\right)= \\
& =\frac{\bar{q}_{\tau}}{\prod_{i=1}^{p} \bar{q}_{\tau-i}^{\phi_{\tau, i}}} \cdot\left[\psi \cdot \prod_{i}^{p} \bar{q}_{t-i} \phi_{\tau, i}\right]= \\
& =\psi_{\tau, i} \cdot \bar{q}_{\tau} \\
\kappa_{\tau}= & q_{t}^{\mathrm{nl}}\left(\bar{q}_{t-i}, \bar{\xi}_{t-j}\right)-\sum_{i=1}^{p} \frac{\partial q_{t}^{\mathrm{nl}}}{\partial q_{t-i}}\left(\bar{q}_{t-i}, \bar{\xi}_{t-j}\right) \cdot \bar{q}_{t-i}-\sum_{j=1}^{q} \frac{\partial q_{t}^{\mathrm{nl}}}{\partial \xi_{t-j}}\left(\bar{q}_{t-i}, \bar{\xi}_{t-j}\right) \cdot \bar{\xi}_{t-j}= \\
= & \bar{q}_{\tau} \cdot\left(1-\sum_{i=1}^{p} \phi_{\tau, i}-\sum_{j=1}^{q} \psi_{\tau, i}\right) \\
& (1)
\end{aligned}
$$

\section{Appendix B: Energy objective linearization}

Energy function, from Equation 17, is written in Equation (B1) as function of problem variables. 


$$
E_{t}=\eta \cdot \Delta k(\tau) \cdot\left[R_{1}\left(v_{t}\right)-R_{2}\left(r_{\mathrm{turb}, t}+r_{\mathrm{spill}, t}\right)\right] \cdot r_{\mathrm{turb}, t}
$$

Equations (B2) are the partial derivatives of Equation (B1).

$$
\begin{gathered}
\frac{\partial E_{t}}{\partial v_{t}}=\eta \cdot \Delta k(\tau) \cdot R_{1}{ }^{\prime}\left(v_{t}\right) \cdot r_{\text {turb }, t} \\
\frac{\partial E_{t}}{\partial r_{\text {turb }, t}}=\eta \cdot \Delta k(\tau) \cdot\left[R_{1}\left(v_{t}\right)-\right. \\
\left.R_{2}{ }^{\prime}\left(r_{\text {turb }, t}+r_{\text {spill }, t}\right) \cdot r_{\text {turb }, t}-R_{2}\left(r_{\text {turb }, t}+r_{\text {spill }, t}\right)\right] \\
\frac{\partial E_{t}}{\partial r_{\text {spill }, t}}=-\eta \cdot \Delta k(\tau) \cdot R_{2}{ }^{\prime}\left(r_{\text {turb }, t}+r_{\text {spill }, t}\right)
\end{gathered}
$$

In Equations (B3), we consider a cylindrical reservoir in proximity of the operational reservoir water level, $h_{0}$, and a linear rating curve in proximity of discharges $r_{\text {turb }, 0}+r_{\text {spill, } 0}$.

$$
\begin{aligned}
R_{1}\left(v_{t}\right) & \approx h_{0}+\frac{1}{A_{0}} \cdot\left(v_{t}-v_{0}\right) \\
R_{2}\left(r_{\text {turb }, t}+r_{\text {spill }, t}\right) & \approx h_{0}^{v}+m_{v}\left(r_{\text {turb }, t}-r_{\text {turb }, 0}+r_{\text {spill }, t}-r_{\text {spill }, 0}\right)
\end{aligned}
$$




$$
\begin{gathered}
E_{t} \approx E_{t}\left(v_{0}, r_{\text {turb }, 0}, r_{\text {spill }, 0}\right)+ \\
+\frac{\partial E_{t}}{\partial v_{t}} \cdot\left(v_{t}-v_{0}\right)+ \\
+\frac{\partial E_{t}}{\partial r_{\text {turb }, t}} \cdot\left(r_{\text {turb }, t}-r_{\text {turb }, 0}\right)+ \\
\quad+\frac{\partial E_{t}}{\partial r_{\text {spill }, t}}\left(r_{\text {spill }, t}-r_{\text {spill }, 0}\right)= \\
=\eta \cdot \Delta k(\tau) \cdot\left\{\left[h_{0}-h_{0}^{v}\right] \cdot r_{\text {turb }, 0}+\right. \\
+\left[\frac{r_{\text {turb }, 0}}{A_{0}}\right] \cdot\left(v_{t}-v_{0}\right)+ \\
+\left[h_{0}-h_{0}^{v}-m_{v}\left(1+r_{\text {turb }, 0}\right)\right]\left(r_{\text {turb }, t}-r_{\text {turb }, 0}\right) \\
\left.+\left[-m_{v}\right]\left(r_{\text {spill }, t}-r_{\text {spill }, 0}\right)\right\}
\end{gathered}
$$

\section{Appendix C: List of main variables}

We use here the classic convention of representing vectors in bold.

$\mathbf{v}_{t}$ Reservoir volumes $\left[m^{3}\right]$

$\mathbf{r}_{t}$ Discharge decision $\left[\mathrm{m}^{3} / \mathrm{s}\right]$

$\mathbf{q}_{t}$ (Flow) scenarios $\left[\mathrm{m}^{3} / \mathrm{s}\right]$

$N_{\text {res }}$ Number of reservoirs [-]

$N_{\text {dec }}$ Number of discharge decisions [-]

$N_{\text {scen }}$ Number of scenarios [-]

$I$ Input matrix $[-]$

$O$ Output matrix $[-]$ 
$\mathbf{c}_{t}(\cdot)$ Inequality constraints $[-]$

$g(\cdot)$ Time-step objective function $[-]$

$F(\cdot)$ Cost-to-go function $[-]$

$\mathcal{F}(\cdot)$ cost-to-go function approximation by Bender's cuts $[-]$

$\mathbb{E}$ Expected value $[-]$

$f_{\mathbf{Q}_{t}}\left(\mathbf{q}_{t}\right)$; Probability density function of $q_{t}[-]$

$\alpha_{\tau, i}, \phi_{\tau, i}, \psi_{\tau, i}, \sigma_{\tau}^{2}$ Periodic ARMA parameters

$\rho_{\tau, i}, \omega_{\tau, i}, \kappa_{\tau}$ Linearized multiplicative model parameters

$\bar{q}_{\tau}$ Climatic average of $Q_{\tau} \mathrm{m}^{3} / \mathrm{s}$

$t$ Time-step index $[-]$

$\tau$ Periodic time-step index $[-]$

$\Delta t$ Daily time-step length [86400s]

$\Delta k$ Number of daily time-steps $[$ days $]$

$T$ Period length $[-]$

$H$ Optimization horizon $[-]$

$e_{t}$ Error due to model linearization $\left[\mathrm{m}^{3} / \mathrm{s}\right]$

$C V$ Cumulative variance $[-]$

$v_{\min }$ Minimum reservoir volume $\left[3.9 \times 10^{9} \mathrm{~m}^{3}\right]$

$v_{\max }$ Maximum reservoir volume $\left[1.5 \times 10^{10} \mathrm{~m}^{3}\right]$

$v_{\text {safety }}$ Reservoir volume safety limit $\left[1.18 \times 10^{10} \mathrm{~m}^{3}\right]$

$r_{\text {spill,max }}$ Maximum discharge through turbines $\left[500 \mathrm{~m}^{3} / \mathrm{s}\right]$

$J_{E}$ Annual average energy production $[G W h]$ 
Author-produced version of the article published in Journal of Irrigation and Drainage Engineering-ASCE, 2017, N¹43(4)

The original publication is available at http://ascelibrary.org

Doi: 10.1061/(ASCE)WR.1943-5452.00007466

RASO ET AL.: EFFECTIVE STREAMFLOW PROCESS MODEL FOR SDDP

\section{References}

$E$ Daily energy production $[M W h]$

$E^{\mathrm{op}}$ Linearized energy production objective $[M W h]$

$v_{0}$ Operational volume $\left[1.18 \times 10^{10} \mathrm{~m}^{3}\right]$

$r_{\text {turb,0 }}$ Operational release through turbines $\left[270 \mathrm{~m}^{3} / \mathrm{s}\right]$

$r_{\text {spill,0 }}$ Operational release through spillages $\left[0 \mathrm{~m}^{3} / \mathrm{s}\right]$

Acknowledgments. Luciano Raso's research is funded by the AXA Research Fund.

Albergel, J., J.-C. Bader, and J.-P. Lamagat, Flood and drought: application to the senegal river management, IAHS Publications-Series of Proceedings and Reports-Intern Assoc Hydrological Sciences, 240, 509-518, 1997.

Arjoon, D., Y. Mohamed, Q. Goor, and A. Tilmant, Hydro-economic risk assessment in the eastern nile river basin, Water Resources and Economics, 8, 16-31, 2014.

Bader, J.-C., J.-P. Lamagat, and N. Guiguen, Gestion du barrage de manantali sur le fleuve sénégal: analyse quantitative d'un conflit d'objectifs, Hydrological sciences journal, 48(4), 525-538, 2003.

Bartolini, P., and J. D. Salas, Modeling of streamflow processes at different time scales, Water resources research, 29(8), 2573-2587, 1993.

Bartolini, P., J. D. Salas, and J. Obeysekera, Multivariate periodic arma $(1,1)$ processes, Water Resources Research, 24(8), 1237-1246, 1988.

Bellman, R. E., and S. E. Dreyfus, Applied dynamic programming, vol. 7962, Princeton University Press, 1966. 
Author-produced version of the article published in Journal of Irrigation and Drainage Engineering-ASCE, 2017, N¹43(4)

The original publication is available at http://ascelibrary.org

Bertsekas, D. P., and J. N. Tsitsiklis, Neuro-dynamic programming: an overview, in Decision and Control, 1995., Proceedings of the 34th IEEE Conference on, vol. 1, pp. 560-564, IEEE, 1995.

Bezerra, B., Á. Veiga, L. A. Barroso, and M. Pereira, Assessment of parameter uncertainty in autoregressive streamflow models for stochastic long-term hydrothermal scheduling, in Power and Energy Society General Meeting, 2012 IEEE, pp. 1-8, IEEE, 2012.

Birge, J. R., and F. Louveaux, Introduction to stochastic programming, Springer Verlag, 1997.

Box, G., G. Jenkins, and G. Reinsel, Time series analysis, Holden-day San Francisco, 1970.

Camacho, F., A. McLeod, and K. Hipel, Multivariate contemporaneous arma model with hydrological applications, Stochastic Hydrology and Hydraulics, 1(2), 141-154, 1987.

Castelletti, A., F. Pianosi, and R. Soncini-Sessa, Water reservoir control under economic, social and environmental constraints, Automatica, 44(6), 1595-1607, doi: 10.1016/j.automatica.2008.03.003, 2008.

Dekking, M., A modern introduction to probability and statistics: understanding why and how, Springer Verlag, 2005.

Fraval, P., J. Bader, L. Mané, H. David-Benz, J. Lamagat, and O. D. Diagne, The quest for integrated and sustainable water management in the senegal river valley, in 5th InterRegional Conference on Environment and Water ENVIROWATER, pp. 5-8, 2002.

Gjelsvik, A., B. Mo, and A. Haugstad, Long-and medium-term operations planning and stochastic modelling in hydro-dominated power systems based on stochastic dual dynamic programming, in Handbook of Power Systems I, pp. 33-55, Springer, 2010. 
Goor, Q., R. Kelman, and A. Tilmant, Optimal multipurpose-multireservoir operation model with variable productivity of hydropower plants, Journal of Water Resources Planning and Management, 137(3), 258-267, 2010.

Hipel, K. W., and A. I. McLeod, Time series modelling of water resources and environmental systems, Elsevier, 1994.

Karamouz, M., F. Szidarovszky, and B. Zahraie, Water resources systems analysis, CRC Press, 2003.

Koutsoyiannis, D., A random walk on water, Hydrology and Earth System Sciences Discussions, 6, 6611-6658, 2009

Linowsky, K., and A. B. Philpott, On the convergence of sampling-based decomposition algorithms for multistage stochastic programs, Journal of optimization theory and applications, 125(2), 349-366, 2005.

Loucks, D. P., Water resource systems models: their role in planning, Journal of Water Resources Planning and Management, 118(3), 214-223, 1992.

Marques, G. F., and A. Tilmant, The economic value of coordination in large-scale multireservoir systems: The parana river case, Water Resources Research, 49(11), 7546$7557,2013$.

Mayne, D. Q., J. B. Rawlings, C. V. Rao, and P. O. M. Scokaert, Constrained model predictive control: Stability and optimality, AUTOMATICA-OXFORD-, 36, 789-814, doi:10.1016/S0005-1098(99)00214-9, 2000.

Nicklow, J., et al., State of the art for genetic algorithms and beyond in water resources planning and management, Journal of Water Resources Planning and Management, $136(4), 412-432,2009$. 
Author-produced version of the article published in Journal of Irrigation and Drainage Engineering-ASCE, 2017, N¹43(4)

The original publication is available at http://ascelibrary.org

$\mathrm{X}-32$ RASO ET AL.: EFFECTIVE STREAMFLOW PROCESS MODEL FOR SDDP

Pereira, M., and L. M. Pinto, Multi-stage stochastic optimization applied to energy planning, Mathematical Programming, 52(1), 359-375, doi:10.1007/BF01582895, 1991.

Pereira, M., G. Oliveira, C. Costa, and J. Kelman, Stochastic streamflow models for hydroelectric systems, Water Resources Research, 20(3), 379-390, 1984.

Philpott, A. B., and Z. Guan, On the convergence of stochastic dual dynamic programming and related methods, Operations Research Letters, 36(4), 450-455, 2008.

Rabiner, L. R., and B.-H. Juang, An introduction to hidden markov models, ASSP Magazine, IEEE, 3(1), 4-16, 1986.

Raso, L., D. Schwanenberg, N. van de Giesen, and P. van Overloop, Short-term optimal operation of water systems using ensemble forecasts, Advances in Water Resources, 71, 200-208, 2014

Reed, P. M., D. Hadka, J. D. Herman, J. R. Kasprzyk, and J. B. Kollat, Evolutionary multiobjective optimization in water resources: The past, present, and future, Advances in Water Resources, 51, 438-456, 2013.

Salas, J. D., Applied modeling of hydrologic time series, Water Resources Publication, 1980.

Salas, J. D., G. Q. Tabios, and P. Bartolini, Approaches to multivariate modeling of water resources time series1, JAWRA Journal of the American Water Resources Association, $21(4), 683-708,1985$.

Seifi, A., and K. W. Hipel, Interior-point method for reservoir operation with stochastic inflows, Journal of water resources planning and management, 127(1), 48-57, 2001.

Shapiro, A., Analysis of stochastic dual dynamic programming method, European Journal of Operational Research, 209(1), 63-72, 2011. 
Shapiro, A., and P. R. Andrzej, Stochastic programming, Elsevier, 2003.

Soncini-Sessa, R., A. Castelletti, and E. Weber, Integrated and participatory water resources management, Elsevier Science, 2007.

Stedinger, J. R., and M. R. Taylor, Synthetic streamflow generation: 1. model verification and validation, Water resources research, 18(4), 909-918, 1982.

Stedinger, J. R., B. F. Sule, and D. P. Loucks, Stochastic dynamic programming models for reservoir operation optimization, Water Resources Research, 20(11), 1499-1505, doi:10.1029/WR020i011p01499, 1984.

Tilmant, A., and R. Kelman, A stochastic approach to analyze trade-offs and risks associated with large-scale water resources systems, Water resources research, 43(6), 2007.

Tilmant, A., and W. Kinzelbach, The cost of noncooperation in international river basins, Water Resources Research, 48(1), 2012.

Tilmant, A., J. Lettany, and R. Kelman, Hydrological risk assessment in the euphratestigris river basin: A stochastic dual dynamic programming approach, Water International, 32(2), 294-309, 2007.

Tilmant, A., D. Pinte, and Q. Goor, Assessing marginal water values in multipurpose multireservoir systems via stochastic programming, Water Resources Research, 44(12), W12,431, doi:10.1029/2008WR007024, 2008.

Tilmant, A., Q. Goor, and D. Pinte, Agricultural-to-hydropower water transfers: sharing water and benefits in hydropower-irrigation systems, Hydrology and Earth System Sciences, 13(7), 1091-1101, 2009.

Tilmant, A., L. Beevers, and B. Muyunda, Restoring a flow regime through the coordinated operation of a multireservoir system: The case of the zambezi river basin, Water 
Author-produced version of the article published in Journal of Irrigation and Drainage Engineering-ASCE, 2017, №143(4)

The original publication is available at http://ascelibrary.org

Resources Research, 46(7), 2010.

Tilmant, A., W. Kinzelbach, D. Juizo, L. Beevers, D. Senn, and C. Casarotto, Economic valuation of benefits and costs associated with the coordinated development and management of the zambezi river basin, Water Policy, 14(3), 490, 2012.

Trezos, T., and W. W.-G. Yeh, Use of stochastic dynamic programming for reservoir management, Water Resources Research, 23(6), 983-996, doi:10.1029/WR023i006p00983, 1987.

Tsitsiklis, J. N., and B. Van Roy, Feature-based methods for large scale dynamic programming, Machine Learning, 22(1-3), 59-94, 1996.

Turgeon, A., Optimal operation of multireservoir power systems with stochastic inflows, Water Resources Research, 16(2), 275-283, doi:10.1029/WR016i002p00275, 1980.

van Overloop, P. J., S. Weijs, and S. Dijkstra, Multiple model predictive control on a drainage canal system, Control Engineering Practice, 16(5), 531-540, doi: 10.1016/j.conengprac.2007.06.002, 2008.

Vecchia, A., Periodic autoregressive-moving average (parma) modeling with applications to water resources1, JAWRA Journal of the American Water Resources Association, $21(5), 721-730,1985$.

Weijs, S. V., Information theory for risk-based water system operation, Ph.D. thesis, Delft University of Technology, 2011. 


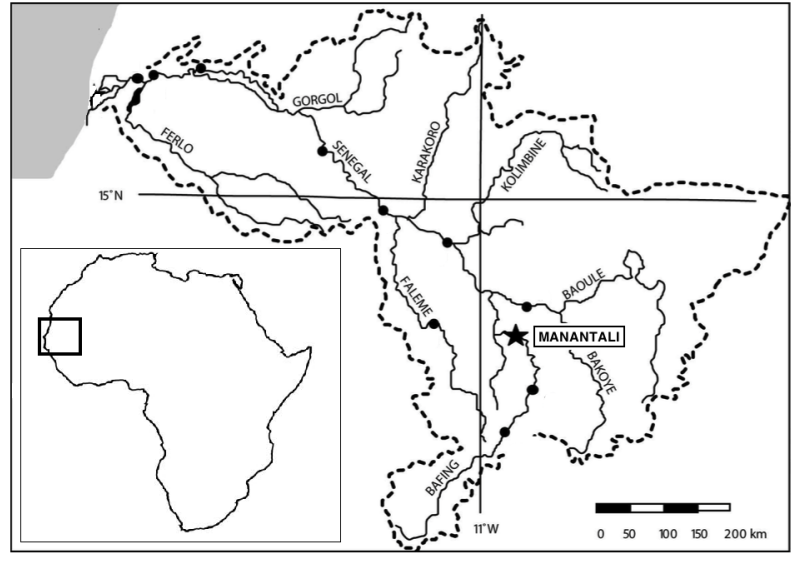

Figure 1. Map of the Senegal Basin.

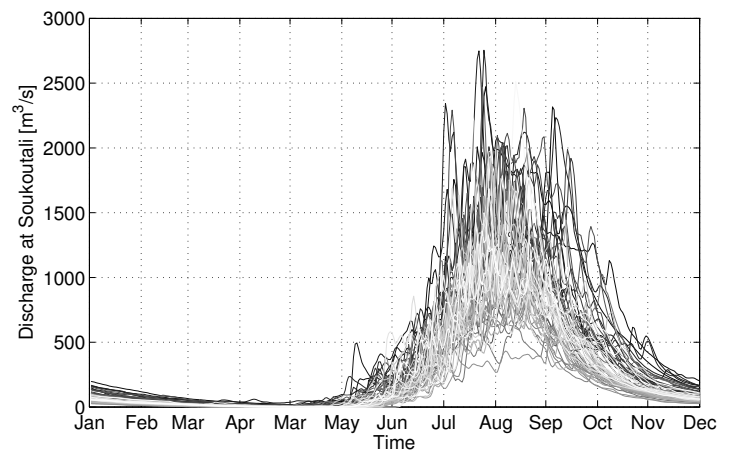

Figure 2. $\quad$ Inflow at Soukoutali from 1 January 1950 to 31 December 2013, daily time-step. 


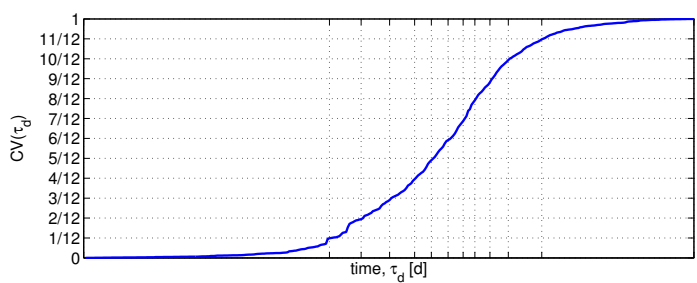

(a) $\tau_{d}$ between 1 and 365

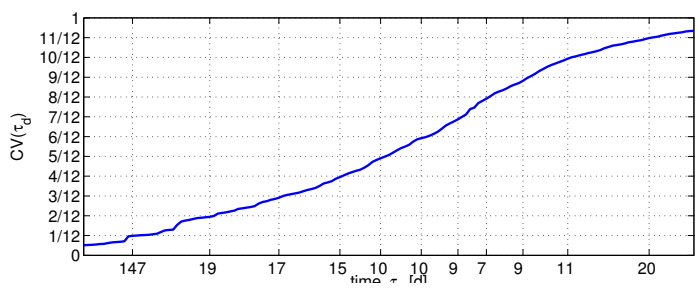

(b) $\tau_{d}$ between 135 and 285

Figure 3. Cumulative Variance on $\tau_{d}$.

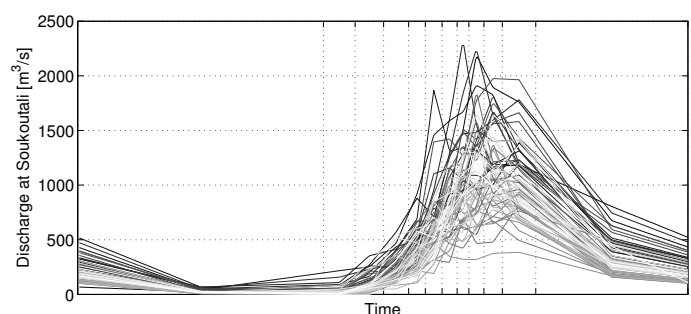

(a) Non-uniform aggregation

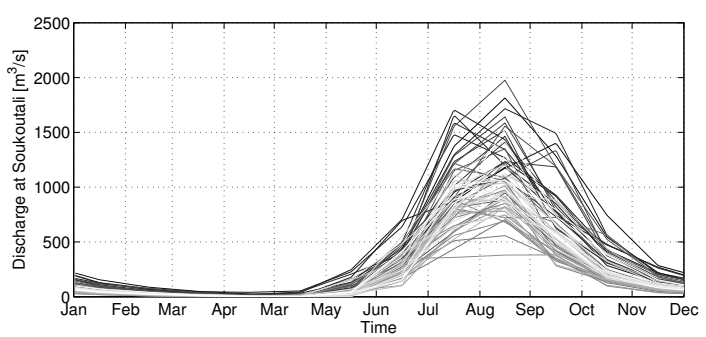

(b) Monthly aggregation

Figure 4. Inflow at Soukoutali from 1 January 1950 to 31 December 2013, aggregated observed discharge. 


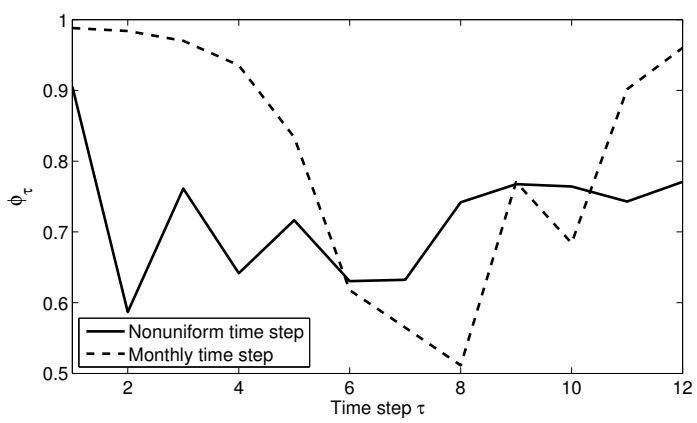

Figure 5. $\phi_{\tau}$ for non-uniform and monthly time-step

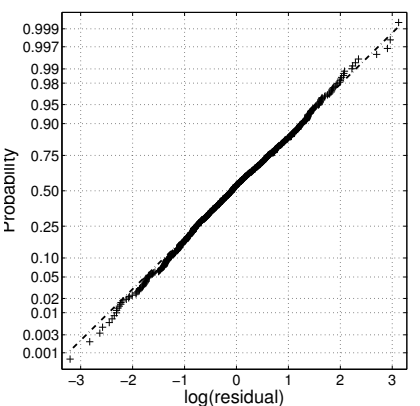

(a)

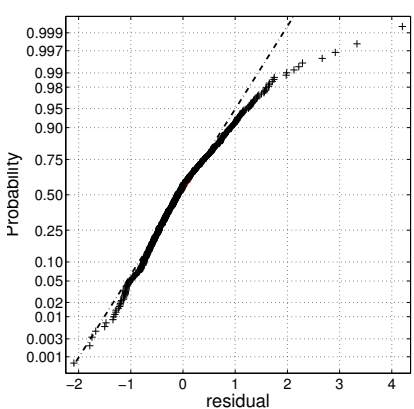

(b)

Figure 6. Normality plot for logarithms of model residuals of Model (9), Multiplicative Model (a), and residuals of Thomas-Fiering, Additive Model (b). 


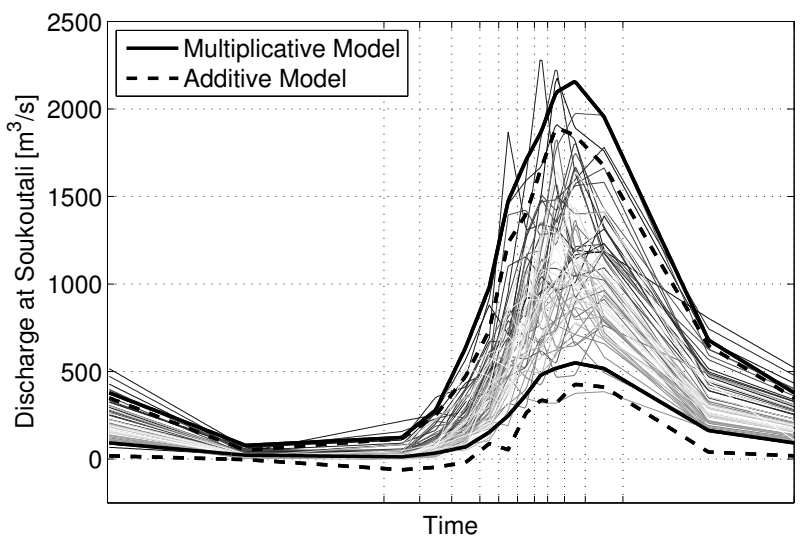

Figure 7. Observed discharge data and 95\% confidence bands for Model (8), or multiplicative model (continuous bold lines) and Thomas-Fiering, or additive model (dashed bold lines).

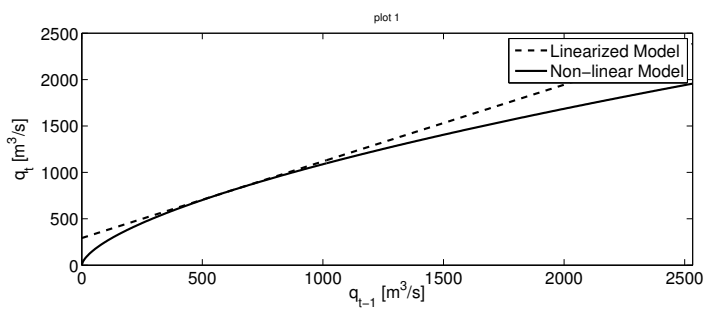

(a) Linear vs non-linear model.

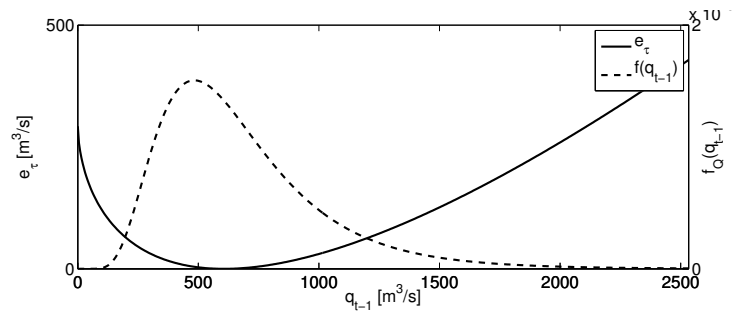

(b) error due to linearisation vs $q_{t-1}$ distribution.

Figure 8. Error due to linearisation at $\tau=7$. 


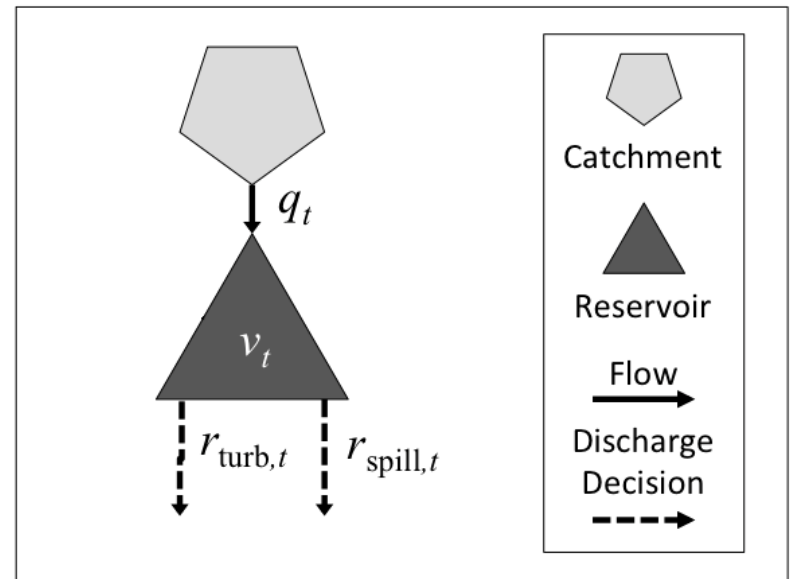

Figure 9. Schema of the Senegal River System at Manantali, including: Manantali reservoir volume, $v_{t}$, inflows at Soukoutali, $q_{t}$, and discharge decisions, $\mathbf{r}_{t}$.

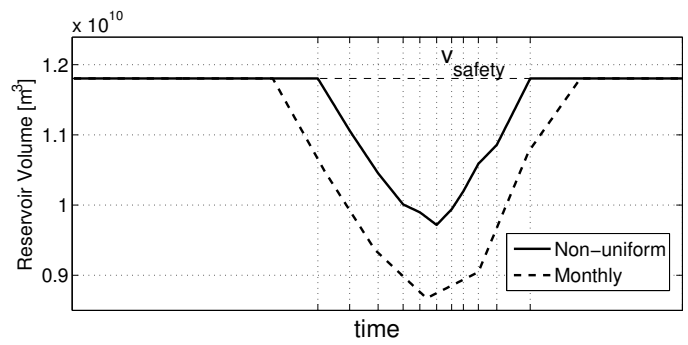

(a)

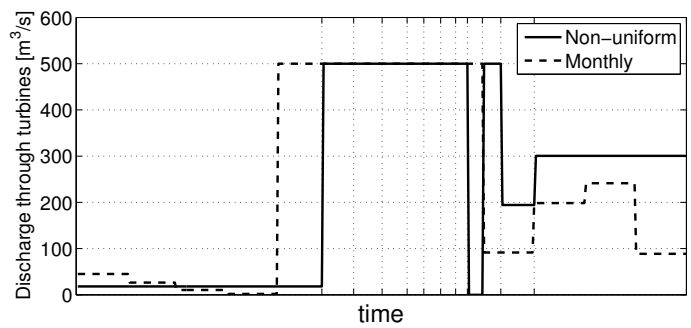

(b)

Figure 10. Reservoir volume (a) and discharge trough turbines (b) for monthly (dashed line) and non-uniform (continuous line) aggregation, year 2005. 